\title{
Thyroid Echography-induced Thyroid Storm and Exacerbation of Acute Heart Failure
}

\author{
Keisuke Nakabayashi, Naomi Nakazawa, Toshiaki Suzuki, Ryotaro Asano, Hideki Saito, \\ Hidekimi Nomura, Daichi Isomura, Hisayuki Okada, Ryo Sugiura and Toshiaki Oka
}

\begin{abstract}
Hyperthyroidism and thyroid storm affect cardiac circulation in some conditions. Several factors including trauma can induce thyroid storms. We herein describe the case of a 57-year-old woman who experienced a thyroid storm and exacerbation of acute heart failure on thyroid echography. She initially demonstrated a good clinical course after medical rate control for atrial fibrillation; however, thyroid echography for evaluating hyperthyroidism led to a thyroid storm and she collapsed. A multidisciplinary approach stabilized her thyroid hormone levels and hemodynamics. Thus, the medical staff should be prepared for a deterioration in the patient's condition during thyroid echography in heart failure patients with hyperthyroidism.
\end{abstract}

Key words: hyperthyroidism, acute heart failure, thyroid echography, thyroid storm

(Intern Med 55: 2209-2212, 2016)

(DOI: 10.2169/internalmedicine.55.6862)

\section{Introduction}

Hyperthyroidism and thyroid storm may impact cardiac circulation in some conditions. Moreover, hyperdynamic states can cause high-output heart failure, while tachycardia causes tachycardia-mediated cardiomyopathy. Therefore, if physicians treat a heart failure patient with hyperthyroidism, they must perform thyroid therapy preceding or simultaneously with heart failure therapy.

A thyroid storm is relatively rare; it is reported in 0.20 people per 100,000 in the Japanese population annually and occurs in $0.22 \%$ of thyrotoxic patients (1). It has a mortality rate of over 10\% in Japan (1), and it is induced by an acute event such as trauma, thyroid or non-thyroid surgery, infection, acute iodine load, or drug provocation. We herein describe a case where mechanical stimulation by thyroid echography induced a thyroid storm, which thus resulted in an exacerbation of acute heart failure.

\section{Case Report}

A 57-year-old woman working in the cleaning department presented with worsening dyspnea and general fatigue. Her medical and family history was unremarkable. She had never experienced pathological weight loss, sweating, tremors, diarrhea, nausea, or vomiting. She was alert and her blood pressure was $120 / 86 \mathrm{mmHg}$, heart rate was $206 / \mathrm{min}$, body temperature was $36.1^{\circ} \mathrm{C}$, respiratory rate was $30 / \mathrm{min}$, and saturation was $96 \%$ (room air). Coarse crackles in all lung fields, bowel sound acceleration, and pretibial edema were detected, however, no goiter or exophthalmic or finger tremors were observed. The electrocardiogram showed an atrial fibrillation rhythm with a rapid ventricular response (heart rate 223/min), ST depression in leads V4-6, high voltage in leads V5-6, and inverted T in leads II, III, and the augmented vector foot lead (Fig. 1, left panel). The chest radiograph indicated pulmonary edema, enlargement of the cardiac silhouette and a cardiothoracic ratio of $64 \%$ (Fig. 1, right panel). Transthoracic echocardiography showed an almost completely preserved ejection fraction, enlargement of all cardiac chambers, moderate mitral regurgitation, moderate tricuspid regurgitation, and an inferior vena cava measuring $30 \mathrm{~mm}$ in diameter. Laboratory data showed the following values: $1.3 \mathrm{mg} / \mathrm{dL}$ of total bilirubin, $48 \mathrm{U} / \mathrm{L}$ of aspartate aminotransferase, $29 \mathrm{U} / \mathrm{L}$ of alanine aminotransferase, 0.34 $\mathrm{mg} / \mathrm{dL}$ of creatinine, $69 \mathrm{mg} / \mathrm{dL}$ of total cholesterol, and $262.4 \mathrm{pg} / \mathrm{ml}$ of brain natriuretic peptide in the plasma. 


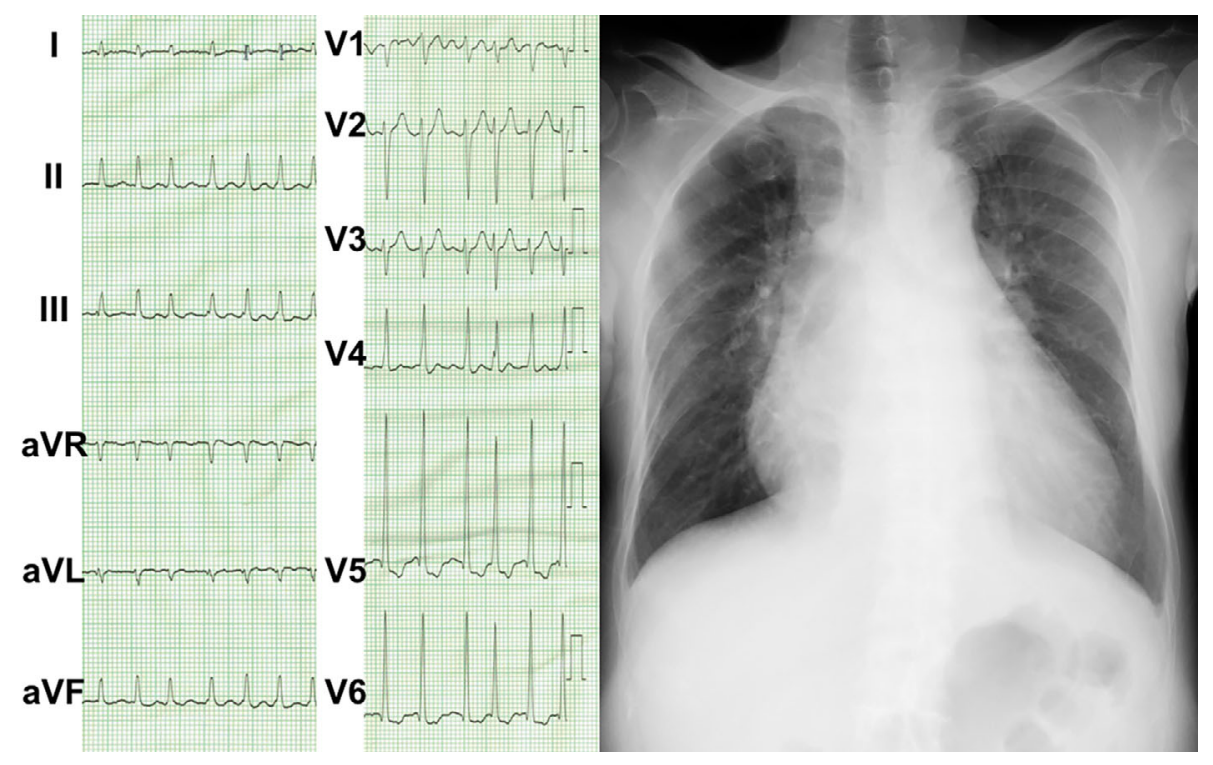

Figure 1. Left panel: an electrocardiogram showing atrial fibrillation rhythm with rapid ventricular response (heart rate 223/min), ST depression in leads V4-6, high voltage in leads V5-6, inverted T in leads II and III, and the augmented vector foot lead. Right panel: a chest radiograph showing pulmonary edema, the enlargement of the cardiac silhouette, and a cardiothoracic ratio of $64 \%$.

According to these findings, we diagnosed the patient with acute decompensated heart failure. The suspected etiologies were as follows: rapid atrial fibrillation, tachycardiamediated cardiomyopathy, diastolic dysfunction, and highoutput heart failure. We first administered medical rate control using intravenous diltiazem (100 mg/day), oral bisoprolol $(2.5 \mathrm{mg} / \mathrm{day})$, and anticoagulation with heparin. This strategy was very successful and decreased the heart rate from 200 to $80 / \mathrm{min}$, relieved the symptoms, and resulted in a body weight loss of $1.5 \mathrm{~kg}$ within 20 hours.

We confirmed that the patient had hyperthyroidism (thyroid stimulating hormone $<0.01 \mathrm{IU} / \mathrm{ml}$, free thyroxine 4.38 $\mathrm{ng} / \mathrm{dL}$, and free triiodothyronine $11.68 \mathrm{pg} / \mathrm{ml}$; thyroid hormone receptor antibody $21.0 \%$; thyroglobulin $163.9 \mathrm{ng} / \mathrm{mL}$; anti-thyroid peroxidase antibody $40 \mathrm{IU} / \mathrm{mL}$; antithyroglobulin antibody $<10 \mathrm{IU} / \mathrm{mL}$ ) on day 2 . We subsequently performed thyroid echography to investigate hyperthyroidism, which revealed a heterogeneous thyroid of normal size and normal vasculature (Fig. 2).

The patient suddenly complained of nausea, thirst, and acute dyspnea. Thereafter, she rapidly lost her orientation and collapsed an hour after undergoing thyroid echography. Her body temperature was $38.4^{\circ} \mathrm{C}$. The chest radiograph showed a butterfly shadow and laboratory data showed hyperlactatemia $(169.5 \mathrm{mg} / \mathrm{dL})$, hyponatremia $(130 \mathrm{mEq} / \mathrm{L})$, hypoglycemia $(40 \mathrm{mg} / \mathrm{dL})$, and hyperbilirubinemia $(5.3 \mathrm{mg} /$ dL total bilirubin and $3.4 \mathrm{mg} / \mathrm{dL}$ direct bilirubin), despite catecholamine support. We suspected a thyroid storm. An emergency administration of $200 \mathrm{mg}$ intravenous hydrocortisone and $15 \mathrm{mg}$ thiamazole was initiated with catecholamine support. The first dose immediately relieved the patient's condition, thus we administered this dosage daily and tapered it gradually thereafter. After confirming the iodine up- take using technetium-99m pertechnetate scintigraphy (Fig. 3) on day 3, we diagnosed the patient with Basedow's disease and the administration of inorganic iodine was also initiated. These multidisciplinary approaches stabilized the patient's thyroid hormone levels (Table) and hemodynamics drastically (Fig. 4). After this acute phase, she recovered with an uneventful course and was discharged on day 22 .

The patient's thyroid and heart rate are currently being controlled in the outpatient clinic with $5 \mathrm{mg}$ of thiamazole and $5 \mathrm{mg}$ of bisoprolol. She has not experienced heart failure for approximately 2 years after the first admission. We obtained the patient's informed consent to publish this case report.

\section{Discussion}

This case is the first published report of thyroid echography-induced thyroid storm and presents two important findings. Firstly, mechanical stress to the thyroid may lead to the exacerbation of acute heart failure in patients with hyperthyroidism. Secondly, the differential diagnosis for the etiology of acute heart failure is important to select the appropriate treatment to rescue the patient.

Mechanical stress to the thyroid may lead to the exacerbation of acute heart failure in patients with hyperthyroidism. Thyroid storms can have many triggers including trauma, thyroid or non-thyroid surgery, infection, acute iodine load, or drug provocation. The trigger in our case may be mechanical compression stress during thyroid echography. Some studies reported that suicide attempts by hanging (2), choking by assailants (3), or blunt trauma (4) resulted in a thyroid storm. In our institution, we observed ventricular fibrillation during thyroid echography in a heart failure pa- 


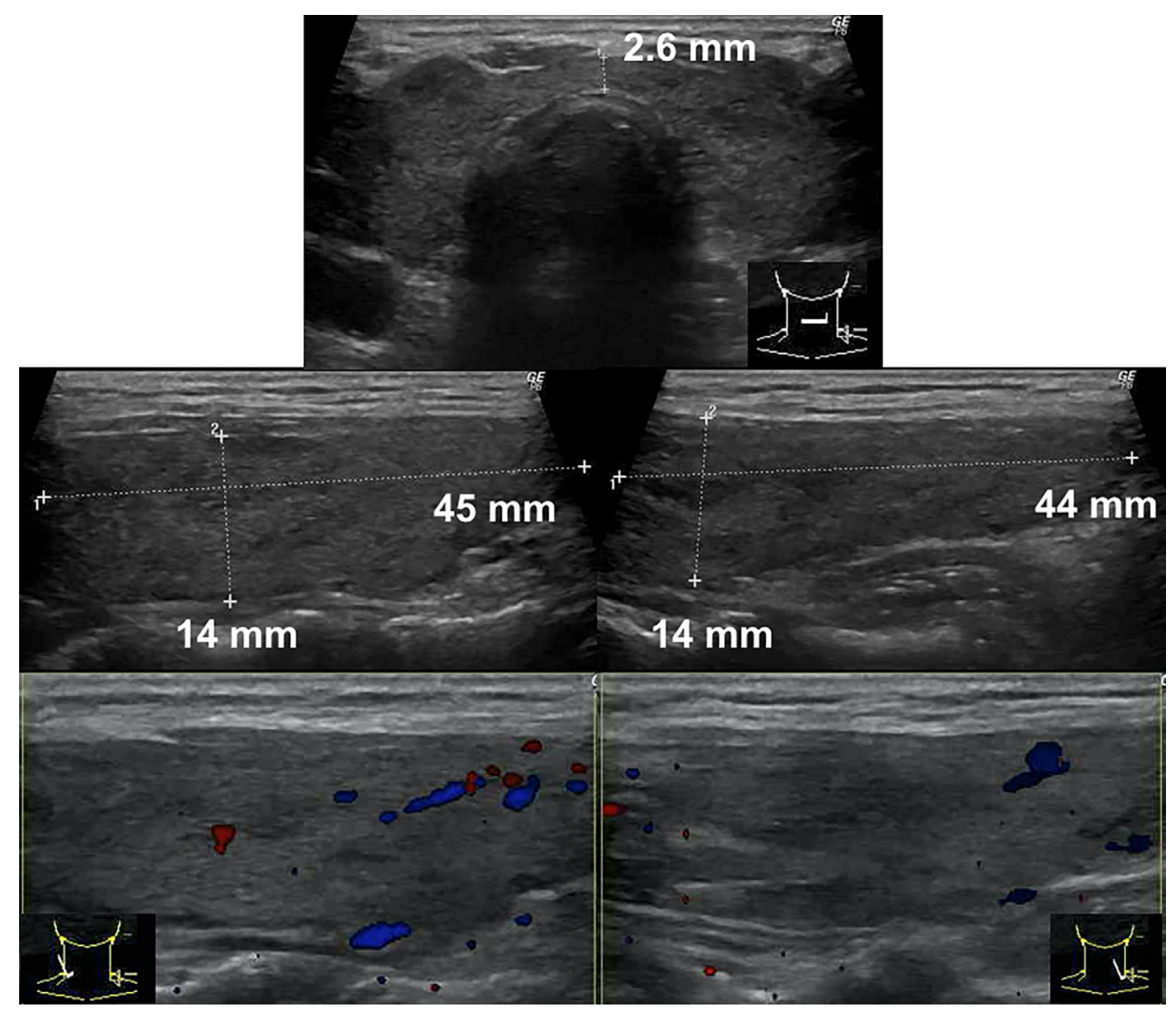

Figure 2. Thyroid echography shows a heterogeneous thyroid of normal size (right lobe $45 \times 14 \mathrm{~mm}$, left lobe $44 \times 14 \mathrm{~mm}$, and isthmus $2.6 \mathrm{~mm}$ ) and normal vasculature.

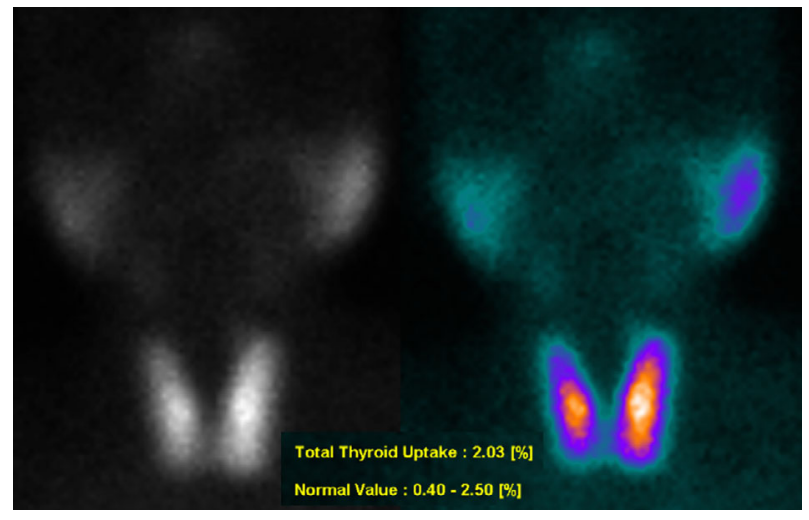

Figure 3. Due to accidental technetium-99m pertechnetate leakage from the injection root, the uptake rate is $2.03 \%$, which is within the normal range. Qualitative imaging shows a higher accumulation in the bilateral thyroid lobes than in the background or salivary glands.

tient with hyperthyroidism. Autopsy findings have suggested that neck trauma induces a thyroid storm through microscopic changes in the thyroid gland, namely, hyperemia and segmental expansion of blood vessels (5). Another study demonstrated that high postmortem levels of free triiodothyronine and thyroglobulin might be indicators of hanging. It appears that applying pressure on the neck region, where the thyroid grand is located, causes the leakage of thyroglobulin as well as free triiodothyronine (6). Our case involved milder neck trauma than other previously reported cases; however, there was a background of hyperthyroidism. Certainly, simple discomfort during thyroid echography might lead to a thyroid storm, however, thyroid echography in acute heart failure patients with hyperthyroidism carries a risk of deterioration.

The differential diagnosis of acute heart failure is important for selecting the appropriate treatment to rescue a patient. We treated the present patient by mainly targeting tachycardia on admission, which was successful. However, rate control therapy and catecholamine were not effective after thyroid echography. We immediately changed the main target etiology of heart failure to a thyroid storm from tachycardia. Although we used a relatively low dose of the treatment in this case, the standard therapy for a thyroid storm can include $20 \mathrm{mg}$ of methimazole every 4 to 6 hours, $200 \mathrm{mg}$ propylthiouracil every 4 hours, 10 drops of Lugol's solution every 6 hours, $100 \mathrm{mg}$ of hydrocortisone every 8 hours, or $4 \mathrm{~g}$ cholestyramine four times daily (7). If we had continued the heart rate-oriented therapy, we might have lost our patient.

Because our institution does not perform laboratory tests 
Table. Thyroid Hormone Levels during the Clinical Course.

\begin{tabular}{cccc}
\hline $\begin{array}{c}\text { Day Post } \\
\text { Admission }\end{array}$ & $\begin{array}{c}\text { TSH } \\
(\mathrm{NR}: 0.35-4.94 \mu \mathrm{IU} / \mathrm{mL})\end{array}$ & $\begin{array}{c}\text { Free Thyroxine } \\
(\mathrm{NR}: 0.7-1.48 \mathrm{ng} / \mathrm{dL})\end{array}$ & $\begin{array}{c}\text { Free Triiodothyronine } \\
(\mathrm{NR}: 1.71-3.71 \mathrm{ng} / \mathrm{dL})\end{array}$ \\
\hline 2 & $<0.01$ & 4.38 & 11.68 \\
4 & $<0.01$ & 2.66 & 4.30 \\
7 & $<0.01$ & 2.08 & 3.18 \\
12 & $<0.01$ & 1.37 & 2.93 \\
\hline
\end{tabular}

TSH: thyroid stimulating hormone, NR: normal range

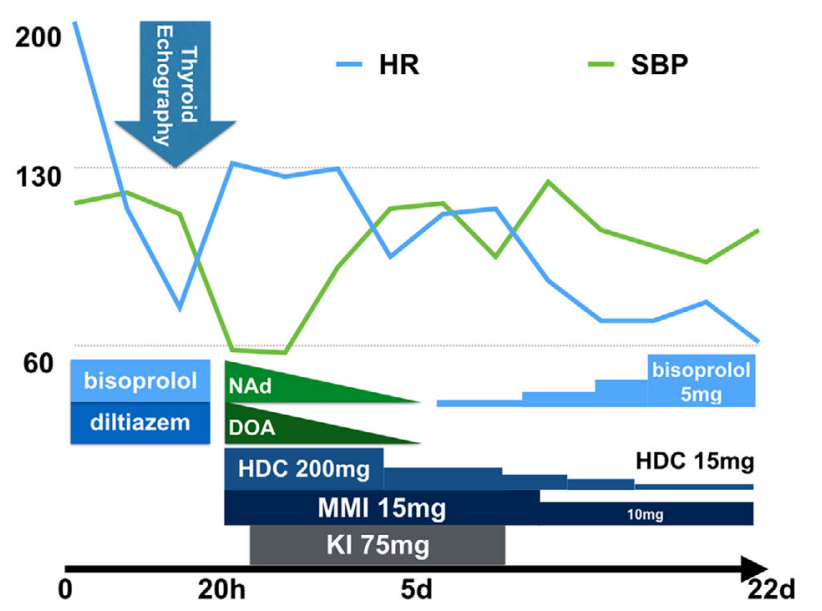

Figure 4. Clinical course. DOA: dopamine, HDC: hydrocortisone, HR: heart rate, KI: potassium iodide, MMI: methimazole, NAd: noradrenaline, SBP: systolic blood pressure

for thyroid measurement within the hospital setting, we obtained a delayed result on day 2. However, we were aware that heart failure and atrial fibrillation with a very rapid ventricular rate are often attributable to hyperthyroidism. Atrial fibrillation, especially in the presence of pre-existent heart disease, can result in clinical heart failure. Such heart failure may be due to an associated rapid ventricular response, which when sustained can lead to tachycardia-mediated cardiomyopathy. The loss of atrial contractile function and decreased diastolic filling time due to tachycardia may cause increased filling pressures, further contributing to this cardi- omyopathy (8).

In conclusion, clinicians should be prepared for a possible deterioration in the patient's condition during thyroid echography for heart failure patients with hyperthyroidism.

The authors state that they have no Conflict of Interest (COI).

\section{References}

1. Akamizu T, Satoh T, Isozaki O, et al. Diagnostic criteria, clinical features, and incidence of thyroid storm based on nationwide surveys. Thyroid 22: 661-679, 2012.

2. Shrum JM, Byers B, Parhar K. Thyroid storm following suicide attempt by hanging. BMJ Case Rep July 9, 2014.

3. Yoshida D. Thyroid storm precipitated by trauma. J Emerg Med 14: 697-701, 1996.

4. Vora NM, Fedok F, Stack BC Jr. Report of a rare case of traumainduced thyroid storm. Ear Nose Throat J 81: 570-572, 574, 2002.

5. Okłota M, Niemcunowicz-Janica A, Dziecioł J, et al. Morphological, microscopic changes in the thyroid gland in the cases of hanging in vertical position with open and closed noose. Arch Med Sadowej Kryminol 61: 331-336, 2011 (in Polish, Abstract in English).

6. Senol E, Demirel B, Akar T, Gülbahar O, Bakar C, Bukan N. The analysis of hormones and enzymes extracted from endocrine glands of the neck region in deaths due to hanging. Am J Forensic Med Pathol 29: 49-54, 2008.

7. Chiha M, Samarasinghe S, Kabaker AS. Thyroid storm: an updated review. J Intensive Care Med 30: 131-140, 2015.

8. Grais IM, Sowers JR. Thyroid and the heart. Am J Med 127: 691698, 2014.

\footnotetext{
(C) 2016 The Japanese Society of Internal Medicine http://www.naika.or.jp/imonline/index.html
} 